\title{
Mask roughness and its implications for LER at the 22- and 16-nm nodes
}

\author{
Patrick P. Naulleau, ${ }^{1}$ Simi A. George, ${ }^{1}$ Brittany M. McClinton ${ }^{2}$ \\ ${ }^{1}$ Center for X-Ray Optics, Lawrence Berkeley National Laboratory, Berkeley, CA 94720 \\ ${ }^{2}$ University of California, Berkeley, CA 94720
}

\begin{abstract}
Line-edge roughness (LER) remains the most significant challenge facing the development of extreme ultraviolet (EUV) resist. The mask, however, has been found to be a significant contributor to image-plane LER. This has long been expected based on modeling and has more recently been demonstrated experimentally. Problems arise from both maskabsorber LER as well as mask multilayer roughness leading to random phase variations in the reflected beam and consequently speckle. Understanding the implications this has on mask requirements for the $22-n m$ half pitch node and below is crucial. Modeling results indicate a replicated surface roughness (RSR) specification of $50 \mathrm{pm}$ and a ruthenium capping layer roughness specification of $440 \mathrm{pm}$. Moreover, modeling indicates that it is crucial to achieve the current ITRS specifications for mask absorber LER which is significantly smaller than current capabilities.
\end{abstract}

Keywords: extreme ultraviolet, lithography, line-edge roughness, multilayer, speckie, photomask, surface roughness

\section{INTRODUCTION}

Line-edge and -width roughness (LER and LWR) remains the most daunting challenge facing the development of commercially viable extreme ultraviolet (EUV) photoresists. LER, however, is not exclusively a resist problem; maskinduced LER can be a significant contributor to the final LER in resist [1-4]. The mask directly contributes to LER in two significant ways with the first and most obvious source being from LER on the mask absorber pattern itself (Fig. 1) which couples to the image plane as described by the LER transfer function (LTF) [1].

The second, and less obvious, source of LER comes from roughness in the clear areas of the mask. This source can be further subdivided into two major categories: roughness replicated through the multilayer stack, and capping layer roughness. As described below, the stack roughness will primarily manifest itself as random phase variations whereas the capping layer roughness will lead to both random phase and random reflectivity with the breakdown depending on the severity of the roughness compared to the total thickness of the capping layer.

We begin by considering the stack roughness which geometrically effects the phase of the reflected beam. Because EUV uses extremely short wavelength light, even sub-nm levels of roughness have significant impact on phase roughness in the reflected field. This reflected field phase roughness at the mask then couples to the intensity variations at the wafer through the concept of speckle $[2,3]$. Being a coherent effect, this mask-induced speckle is highly dependent on illumination coherence and defocus. In order for the roughness on the mask to geometrically couple to phase it must be replicated throughout at least the top 10 layers of the multilayer since EUV reflectivity relies on the Bragg effect. Such roughness is depicted in Fig. 1 and referred to as replicated surface roughness (RSR). Fundamentally, such roughness most typically originates from the substrate and then propagates all the way to the surface of the multilayer through conformal deposition of the layers. It is evident, however, that the multilayer deposition process will not support conformal growth at all frequencies and in practice it becomes a lowpass filtering process with the characteristics of the filtering depending on the details of the deposition method. From this description, it is evident that RSR is not fully equivalent to substrate roughness and that the smoothing characteristics of the multilayer are needed in order to predict RSR given the substrate roughness. A reasonable estimate of the RSR can be obtained through AFM surface characterization immediately following the multilayer deposition process. This assumes the roughness of the deposited layers is insignificant compared to the magnitude of the roughness propagating from the substrate. The validity of this assumption again depends on the characteristics of the coating process. Given an RSR value, the resulting phase roughness can readily be found geometrically as

$$
\text { PhaseRoughness }=\frac{2 \pi}{\lambda} 2 R S R \text {. }
$$


Capping layer roughness, which is not integral to the Bragg structure, affects the phase through refraction instead of reflection, thus the optical properties of the capping layer play an important role in dictating the scaling from surface roughness to induced phase roughness. Moreover, if the magnitude of the roughness becomes significant compared to the total thickness of the capping layer, direct reflectivity variations at the mask could be induced.

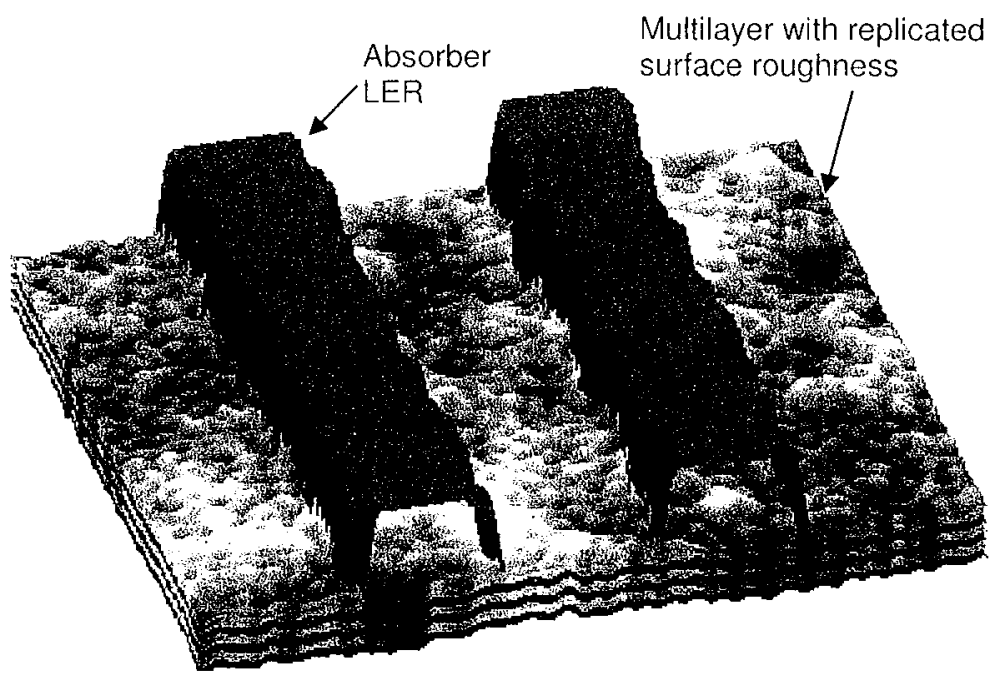

Fig. 1. Schematic depicting the two major mask sources of image-plane LER.

To model a mask with phase roughness we treat it simply as a phase-shift mask. Figure 2 shows clear field modeling results for a mask with $230 \mathrm{pm}$ of RSR and perfectly uniform reflectivity. The images show how the phase roughness is transformed to speckle through the imaging process with the contrast of the speckle increasing both as the coherence and defocus are increased. Other important factors in the determination of the speckle are the correlation length of phase roughness and numerical aperture of the imaging optic. If one imagines a perfect line space structure overlain on this speckle field, it is evident that speckle-induced intensity variations along the length of the line will lead to LER.

\begin{tabular}{|c|c|c|c|}
\hline Conditions & $\begin{array}{c}\mathrm{NA}=0.32 \\
\text { Defocus }=0 \\
\sigma=0.5\end{array}$ & $\begin{array}{c}\mathrm{NA}=0.32 \\
\text { Defocus }=50 \mathrm{~nm} \\
\sigma=0.5\end{array}$ & $\begin{array}{c}\mathrm{NA}=0.32 \\
\text { Defocus }=50 \mathrm{~nm} \\
\sigma=0.3\end{array}$ \\
\hline Contrast & $0.9 \%$ & $6 \%$ & $9 \%$ \\
\hline Aerial image & & 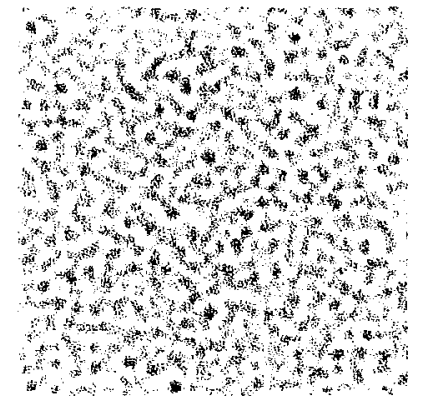 & 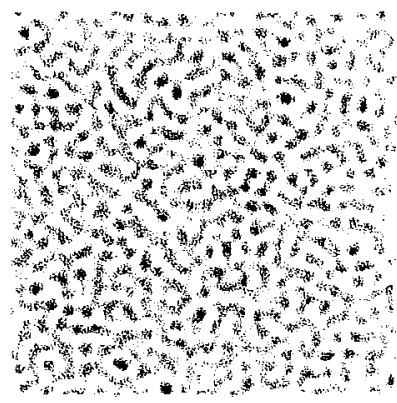 \\
\hline
\end{tabular}

Fig. 2. Clear field modeling results for a mask with 230 pm of RSR under three different optical conditions. Field size is $1 \mu \mathrm{m}$ and the system demagnification is $4 x$. 


\section{MASK-INDUCED LER TARGETS}

In order to achieve our goal of determining mask roughness specifications (surface and absorber) we must first define the acceptable magnitude of mask-induced LER. Because the ITRS [5] defines only the acceptable total LER in resist, we arbitrarily set the mask-induced limit to be a $10 \%$ contribution to the total LER assuming the mask contributions to add in quadrature with all other contributions. Given the ITRS LWR specifications of 1.8 and $1.3 \mathrm{~nm}$ for the 22 and $16-\mathrm{nm}$ half-pitch nodes, respectively, the $10 \%$ mask contribution assumption set the mask-induced LWR limits to be 0.7 and 0.5 $\mathrm{nm}$, respectively.

\section{MASK LER}

We begin by considering the expected image plane contribution from mask LER. For this task we initially assume the mask LER magnitudes to match ITRS specifications (Table 1) and the LER power spectrum to match published results [4]. For the 22-nm half-pitch case, we assume a NA of 0.32 and disk illumination with a $\sigma$ of 0.5 . For 16-nm half pitch, we consider two different optic configurations: first is 0.32 NA and quadrupole illumination with a pole offset of 0.66 and a pole radius of 0.1 , and second is $0.42 \mathrm{NA}$ with annular illumination with an inner $\sigma$ of 0.4 and an outer $\sigma$ of 0.6 . In all cases we assume the optics to be aberration free.

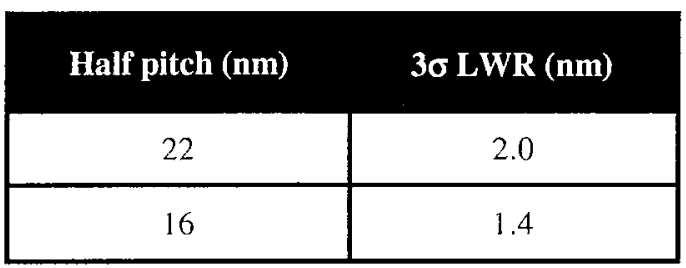

Table 1. ITRS specified mask LWR magnitudes.

Under the assumption described above, Fig. 3 shows the image plane LWR through focus for the three different optical configurations. In general, we see the expected approximately $4 \times$ reduction in LWR. Note that for the resolution enhancing illumination cases which provide improved depth of focus, we observe very little dependence of $L W R$ on defocus. Although the absolute LWR values are small, they are not insignificant when compared to the mask-induced LWR limits of 0.7 and $0.5 \mathrm{~nm}$ for the 22 and 16-nm nodes, respectively. The two different mask sources of LWR, however, can be assumed to be uncorrelated and thus will add in quadrature. Using the average best focus LWR value of $0.32 \mathrm{~nm}$, this amount of mask absorber LER would imply a mask phase roughness induced LWR limit of 0.62 and 0.38 $\mathrm{nm}$ for the 22 and 16-nm half-pitch nodes, respectively. Clearly the mask LER has a significant impact on the allowable mask RSR contribution at the $16-\mathrm{nm}$ node

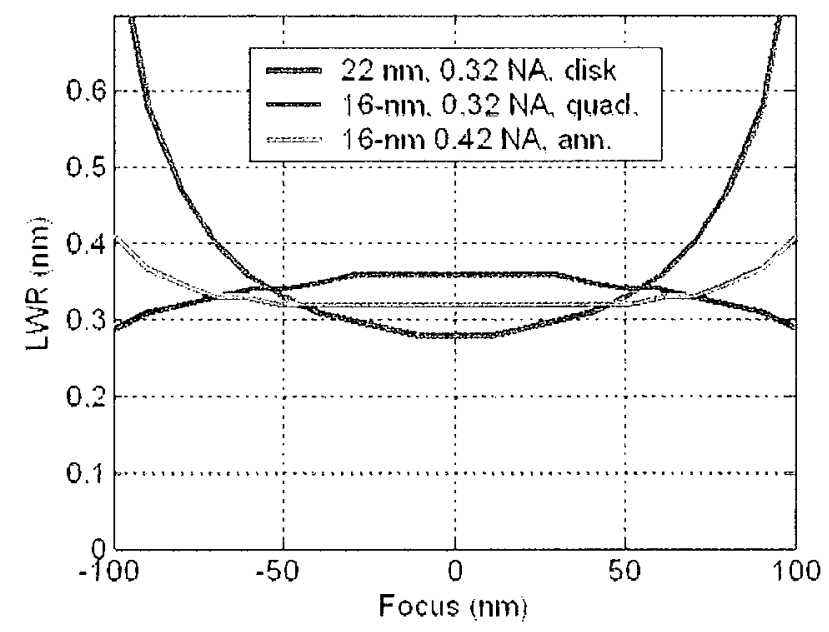

Fig. 3. Computed image-plane LWR as a function of focus for mask absorber LER alone using ITRS mask LWR values. 
It is important to note that the ITRS-specified mask LWR values assumed above are quite far from what is achievable today. To obtain more realistic values we poled a series of both captured and independent mask shops to determine the mask LWR one might expect at the 22 and 16-nm half-pitch nodes (Table 2). These values are on the order of $4 \times$ larger than those specified in the ITRS. Repeating the modeling based on these values yields the results in Fig. 4 . In this case the resulting LWR is larger than our entire mask-induced LWR allocation for both the 22 and 16-nm nodes. Moreover, for the 16-nm node at $0.32 \mathrm{NA}$, the mask LWR contribution exceeds the entire ITRS wafer plane LWR specification. It is evident that significant improvement in mask LWR is needed in order to achieve target wafer plane values.
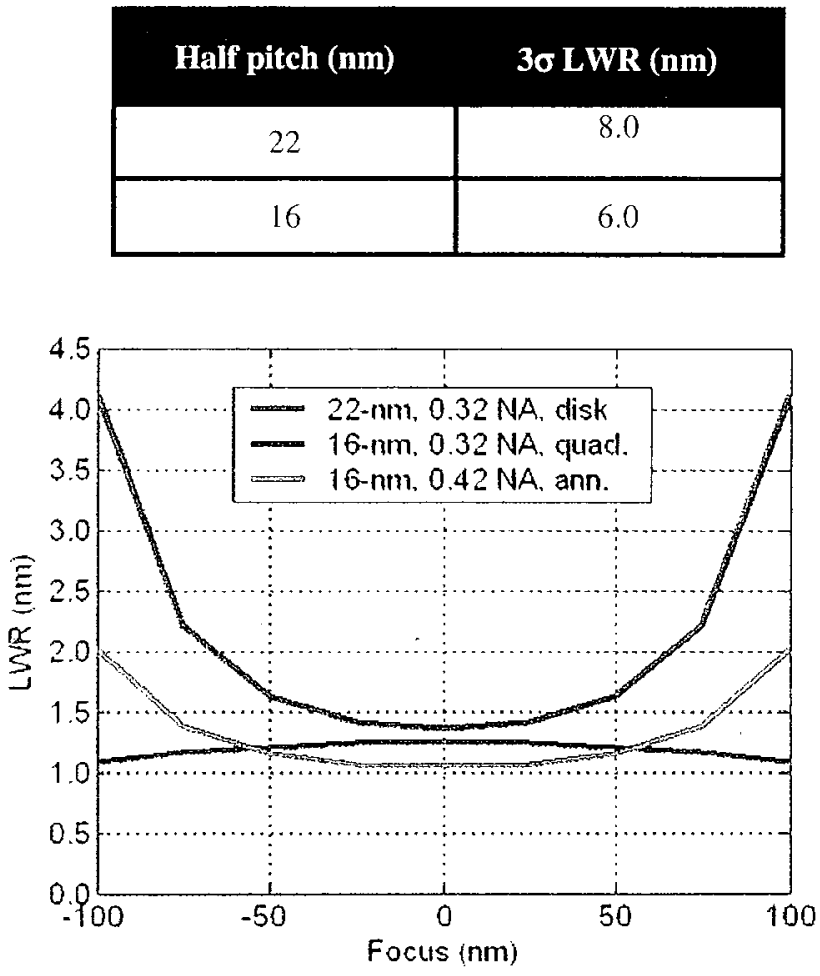

Table 2. Expected LWR magnitudes based on mask shop poling.
Fig. 4. Computed image-plane LWR as a function of focus for mask absorber LER alone using expected mask LWR values based on mask-shop poling.

\section{MASK RSR}

Next we consider the impact of mask RSR. Because RSR-induced LER is highly sensitive to focus and approaches zero at best focus, it is important to evaluate the induced LER at the edge of the target focus window. We set the target focus window based on a normalized image log slope value of 1.5 . Doing so yields focus evaluation points of $80 \mathrm{~nm}, 65 \mathrm{~nm}$, and $35 \mathrm{~nm}$ for the 22-nm $0.32 \mathrm{NA}, 16-\mathrm{nm} 0.32 \mathrm{NA}$, and 16-nm $0.42 \mathrm{NA}$ modes, respectively. Figure 5 shows the induced LWR at the evaluation focus as a function of RSR for the three different configurations. The two 16-nm configurations yield quite similar results. Recalling that the target values for RSR-induced LWR are 0.62 and $0.38 \mathrm{~nm}$ for the 22 and 16-nm half-pitch nodes, respectively, we see that 22-nm node requires an RSR of less than 46 pm, whereas the 16-nm node requires an RSR of approximately $75 \mathrm{pm}$ for both NAs. Note that the 22 -nm node value could readily be relaxed by reducing the target focus window. Note also, that these RSR specifications rely on achieving the extremely stringent mask LWR specification defined in the ITRS. Backing off on those values will simultaneously force a decrease in acceptable RSR values unless the LWR specs themselves are reduced or focus latitude is sacrificed. 


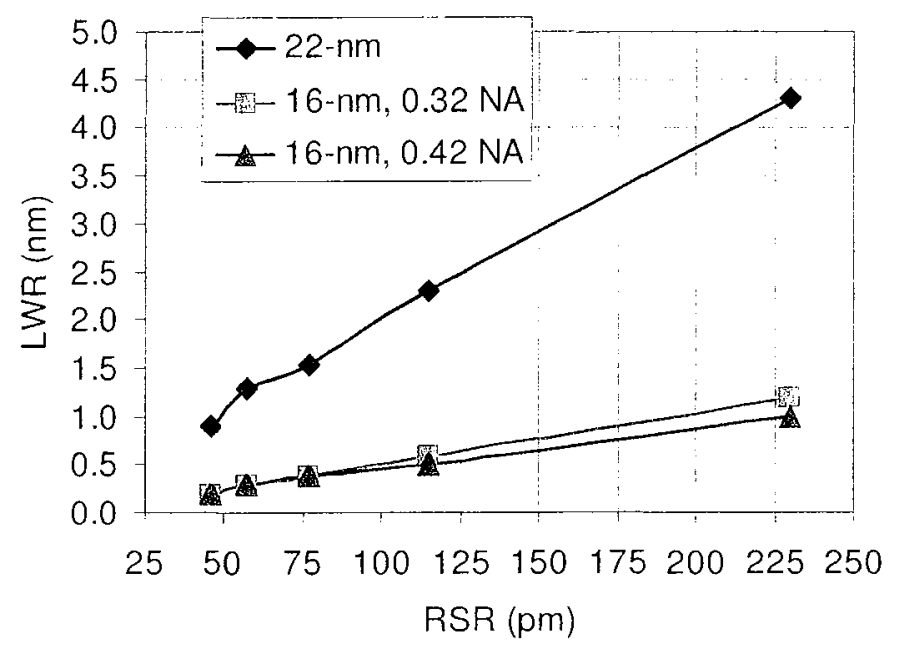

Fig. 5. Mask phase roughness induced LWR at the evaluation focus as a function of RSR for the 22 and 16-nm half-pitch nodes.

\section{CAPPING LAYER ROUGHNESS}

Next we consider the impact of capping layer roughness. As discussed above, optical properties are crucial in determining the impact of capping layer roughness. To illustrate this fact, we consider three different materials: silicon, ruthenium, and carbon. We choose silicon and ruthenium because they are common capping materials and we choose carbon noting that another potential source of surface roughness could be the deposition of carbon onto the mask during operation. In addition to affecting the throughput, if rough, such a layer of carbon might also have the same effects as a rough capping layer. Table 3 shows the double pass phase shift per nm of material as well as the equivalent amount of refractive material roughness required to get the same phase roughness as $50 \mathrm{pm}$ of RSR. If silicon is used, clearly capping layer roughness is not an issue; on the other hand, ruthenium has significant phase shifting properties which makes it susceptible to sub-nm roughness. Carbon also has non-trivial phase shifting properties, yet it is not likely that one would ever get to the point where roughness might affect the LER given the significant throughput loss one would suffer from a nominal thickness that would likely be many times larger than the variation representing the roughness.

\begin{tabular}{|c|c|c|}
\hline Capping Material & $\begin{array}{c}\text { Double Pass Phase Shift } \\
\text { per nm of material }\end{array}$ & $\begin{array}{c}\text { Roughness Equivalent } \\
\text { to 50 pm RSR* }\end{array}$ \\
\hline $\mathrm{Si}$ & $0.002^{\circ}$ & $730 \mathrm{~nm}$ \\
\hline $\mathrm{Ru}$ & $6^{\circ}$ & $0.44 \mathrm{~nm}$ \\
\hline $\mathrm{C}$ & $2^{\circ}$ & $1.25 \mathrm{~nm}$ \\
\hline
\end{tabular}

Table 3. Double pass phase shift per nm of material as well as the equivalent amount of refractive material roughness required to get the same phase roughness as 50 $\mathrm{pm}$ of RSR.

Although both RSR and capping layer roughness lead to induced phase error in the reflected wave, significant differences would be expected in the spatial properties of the two different sources. RSR which is expected to originate from the substrate will have spectral content that is limited by the multilayer conformal growth process. Analysis of a typical EUV mask has shown the RSR correlation length to be approximately $125 \mathrm{~nm}$ [4]. This is much longer than the correlation length one might expect from a rough capping layer that might have been roughened during an etch or cleaning process.

Understanding the relevance of correlation length in the coupling of phase roughness to image plane LER is thus important. Figure 6 shows the image-plane LER as a function of focus for various correlation lengths of roughness. In each case the phase roughness is assumed to be equivalent to $50 \mathrm{pm}$ of RSR and the $22-n \mathrm{~m}$ node case is assumed. The LER is seen to be strongly dependent on correlation length and the effect is not monotonic. To better visualize the nonmonotonic behavior. Fig. 7 shows a plot of the image-plane LER as a function of correlation length at $50 \mathrm{~nm}$ of defocus. 
A strong peak in LER is found at a correlation length of approximately $100 \mathrm{~nm}$ which coincidently matches closely to the correlation length of the RSR from a typical EUV mask. This suggests that as an alternative to driving down the RSR on future masks, effort could also be put into changing the correlation length of the remaining RSR to minimize coupling to LER. We note that is can be shown that the peak in LER as a function of correlation length can be related back to the numerical aperture of the optic. The peak correlation length will be very close to the object-side resolution limit of the projection optics.

Although the RSR correlation length matches closely to the peak susceptibility correlation for the 0.32-NA system, capping layer roughness correlation length would be expected to be significantly shorter, thereby, further reducing the sensitivity to such roughness.

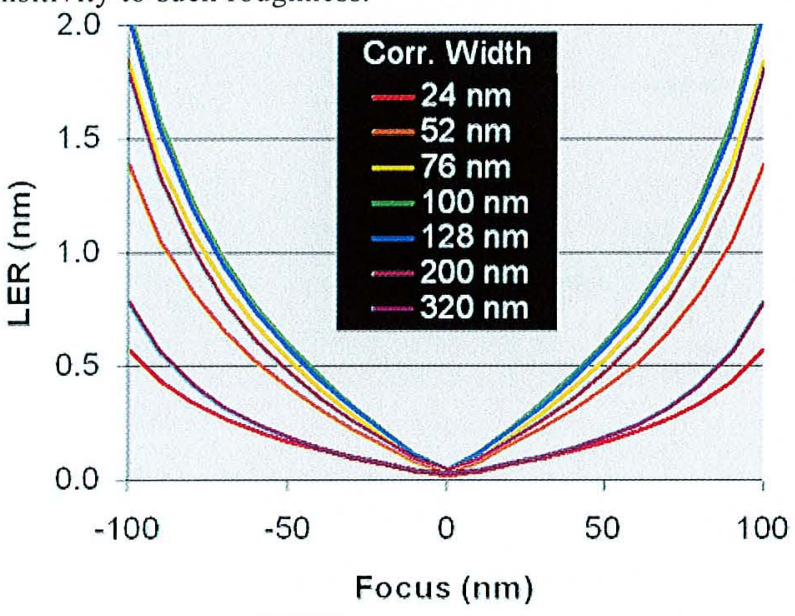

Fig. 6. Image-plane LER as a function of focus for various correlation lengths of roughness. In each case the phase roughness is assumed to be equivalent to $50 \mathrm{pm}$ of RSR and the 22-nm node case is assumed.

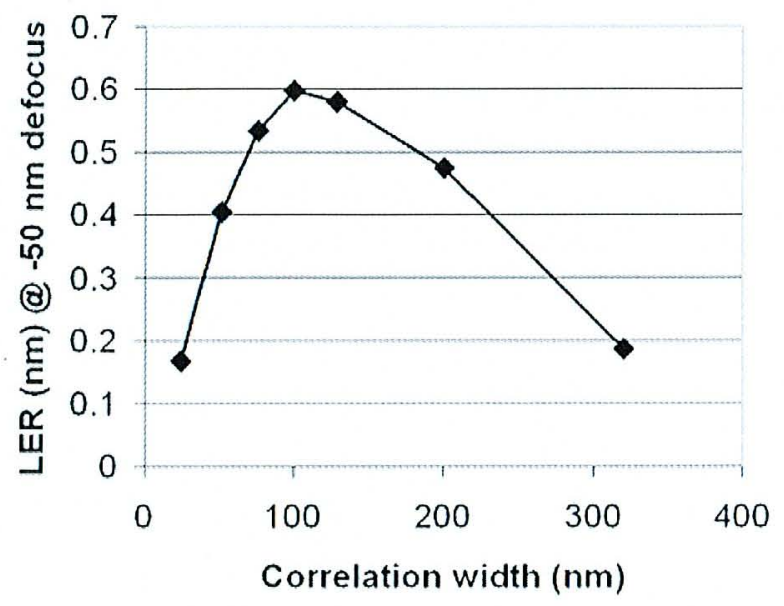

Fig. 7. Image-plane LER as a function of roughness correlation length at $50-\mathrm{nm}$ defocus.

\section{SUMMARY}

Mask effects have been found to play a significant role in image plane LER. Both replicated surface roughness (RSR) and mask absorber LER are crucial. Modeling shows that RSR will need to be on the order of $50 \mathrm{pm}$ or smaller to meet the 22 and 16- $\mathrm{nm}$ node LER targets assuming that current mask absorber LER targets, as defined in the ITRS, are achieved. It is important to note, however, that the ITRS mask LER targets are considerably tighter than what is expected to be achievable. Using LER estimates gathered from various mask makers, the mask LER alone could consume nearly $100 \%$ or more of the total image-plane LER budget. Failure to meet the ITRS targets for mask LER in the future would further stress the mask RSR specifications. Finally, analysis also shows that capping layer roughness could be a concern; however, tolerances for this roughness are nearly an order of magnitude larger than for RSR. Analysis also showed that roughness correlation length is a key factor. This fact will likely even further increase the tolerance to capping layer roughness as the tolerances presented here assumed the capping layer roughness correlation length to match that experimentally measured for the RSR. In practice we expect capping layer roughness correlation length to be much shorter than RSR correlation length. This sensitivity to correlation length also suggests that reduced sensitivity to RSR could be achieved by shifting its value from it current location which is near the peak of susceptibility for a 0.32 -NA optical system.

This work was funded in part by SEMATECH and performed at Lawrence Berkeley National Laboratory using the SEMATECH MET exposure facility at the Advanced Light Source. Lawrence Berkeley National Laboratory's Advanced Light Source synchrotron facility is supported by the Director, Office of Science, of the U.S. Department of Energy under Contract No. DE-AC02-05CH11231. 


\section{REFERENCES}

1. P. Naulleau and G. Gallatin, "The line-edge roughness transfer function and its application to determining mask effects in EUV resist characterization," Appl. Opt. 42. 3390-3397 (2003).

2. N. Beaudry, T. Milster, "Effects of mask roughness and condenser scattering in EUVL systems." Proc. SPIE. 3676. 653-662 (1999).

3. P. Naulleau, "The relevance of mask-roughness-induced printed line-edge roughness in recent and future EUV lithography tests," Appl. Opt. 43, 4025-4032 (2004).

4. P. Naulleau and S. George, "Implications of image plane line-edge roughness requirements on extreme ultraviolet mask specifications," Proc. SPIE 7379, 73790O-737900-11 (2009).

5. http://ww' itrs.nev/ 